\title{
Changing malaria fever test positivity among paediatric admissions to Tororo district hospital, Uganda 2012-2019
}

Arthur Mpimbaza ${ }^{1,2^{*}} \mathbb{D}$, Asadu Sserwanga², Damian Rutazaana ${ }^{3}$, James Kapisi², Richard Walemwa4, Laurissa Suiyanka ${ }^{5}$, David Kyalo ${ }^{5}$, Moses Kamya ${ }^{2}$, Jimmy Opigo ${ }^{3}$ and Robert W. Snow ${ }^{5,6}$

\begin{abstract}
Background: The World Health Organization (WHO) promotes long-lasting insecticidal nets (LLIN) and indoor residual house-spraying (IRS) for malaria control in endemic countries. However, long-term impact data of vector control interventions is rarely measured empirically.

Methods: Surveillance data was collected from paediatric admissions at Tororo district hospital for the period January 2012 to December 2019, during which LLIN and IRS campaigns were implemented in the district. Malaria test positivity rate (TPR) among febrile admissions aged 1 month to 14 years was aggregated at baseline and three intervention periods (first LLIN campaign; Bendiocarb IRS; and Actellic IRS + second LLIN campaign) and compared using before-and-after analysis. Interrupted time-series analysis (ITSA) was used to determine the effect of IRS (Bendiocarb + Actellic) with the second LLIN campaign on monthly TPR compared to the combined baseline and first LLIN campaign periods controlling for age, rainfall, type of malaria test performed. The mean and median ages were examined between intervention intervals and as trend since January 2012.
\end{abstract}

Results: Among 28,049 febrile admissions between January 2012 and December 2019, TPR decreased from 60\% at baseline (January 2012-October 2013) to 31\% during the final period of Actellic IRS and LLIN (June 2016-December 2019). Comparing intervention intervals to the baseline TPR (60.3\%), TPR was higher during the first LLIN period (67.3\%, difference 7.0\%; 95\% Cl 5.2\%, 8.8\%, p<0.001), and lower during the Bendiocarb IRS (43.5\%, difference - 16.8\%; $95 \% \mathrm{Cl}-18.7 \%,-14.9 \%)$ and Actellic IRS (31.3\%, difference $-29.0 \% ; 95 \% \mathrm{Cl}-30.3 \%,-27.6 \%, \mathrm{p}<0.001)$ periods. ITSA confirmed a significant decrease in the level and trend of TPR during the IRS (Bendicarb + Actellic) with the second LLIN period compared to the pre-IRS (baseline + first LLIN) period. The age of children with positive test results significantly increased with time from a mean of 24 months at baseline to 39 months during the final IRS and LLIN period.

Conclusion: IRS can have a dramatic impact on hospital paediatric admissions harbouring malaria infection. The sustained expansion of effective vector control leads to an increase in the age of malaria positive febrile paediatric admissions. However, despite large reductions, malaria test-positive admissions continued to be concentrated in children aged under five years. Despite high coverage of IRS and LLIN, these vector control measures failed to interrupt transmission in Tororo district. Using simple, cost-effective hospital surveillance, it is possible to monitor the public health impacts of IRS in combination with LLIN.

\footnotetext{
${ }^{*}$ Correspondence: arthurwakg@yahoo.com

${ }^{1}$ Child Health and Development Centre, Makerere University, College of Health Sciences, Kampala, Uganda

Full list of author information is available at the end of the article
}

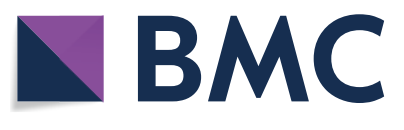

(c) The Author(s) 2020. This article is licensed under a Creative Commons Attribution 4.0 International License, which permits use, sharing, adaptation, distribution and reproduction in any medium or format, as long as you give appropriate credit to the original author(s) and the source, provide a link to the Creative Commons licence, and indicate if changes were made. The images or other third party material in this article are included in the article's Creative Commons licence, unless indicated otherwise in a credit line to the material. If material is not included in the article's Creative Commons licence and your intended use is not permitted by statutory regulation or exceeds the permitted use, you will need to obtain permission directly from the copyright holder. To view a copy of this licence, visit http://creativeco mmons.org/licenses/by/4.0/. The Creative Commons Public Domain Dedication waiver (http://creativecommons.org/publicdomain/ zero/1.0/) applies to the data made available in this article, unless otherwise stated in a credit line to the data. 
Keywords: Changing, Test, Positivity, Paediatric admissions, Uganda

\section{Background}

Indoor Residual Spraying (IRS) has regained attention as a means to prevent malaria infection in high transmission areas of Africa when used in combination with insecticide-treated nets, largely as a result of funding made available by the US President's Malaria initiative (PMI) [1]. Carbamates, organochlorines, organophosphates, pyrethroids, and more recently neonicotinoids, have been used in 23 countries in SSA since 1997, resulting in approximately 21 million people being protected by one form of IRS in 2017 [2].

In Uganda, between 1959-1964, IRS using dichlorodiphenyl-trichloro-ethane (DDT) with three rounds of mass drug administration (MDA) with single doses of chloroquine-pyrimethamine was piloted in Kigezi and parts of Masaka districts [3]. During the first year of the operations parasite prevalence declined from 16.6 to 0.3\% [4]. Between 1963 and 1964, a large-scale field trial of Malathion was carried out in Masaka district reducing vector densities to 0.0011 from 66 per house per day within a year [5]. Following on from these successes, the Uganda malaria pre-eradication programme was established in 1964 as a joint Government of Uganda-WHO project [6]. However, the period between 1970 and 1990 was characterized by a general collapse of the country's health system as a result of civil war and political unrest. During this period, IRS alongside other forms of malaria prevention were largely abandoned.

Lambda-cyhalothrin IRS was introduced in 2004 to tackle localized epidemics in Kabale, Kisoro and Kanungu districts. In 2005, Uganda became one of the first countries in sub-Saharan Africa to benefit from PMI funding, supporting a sustained IRS campaign in different parts of the country. From 2006, IRS with lambda-cyhalothrin was introduced to Kabale and Kanungu districts [7]. PMI provided their implementing partner over US\$ 150 million between 2007-2012 to continue, and expand, IRS activities with lamda-cyhalothrin across districts in the north (Lamwo, Kitgum, Pader, Agogo, Kole, Oyam, Apac, Gulu, Amuru and Nwoya) and Kanungu [8, 9]. For a brief period in 2008, the Ministry of Health used DDT as part of IRS programs in Apac and Oyam districts. In Katakwi and Kumi, in the Teso region, between 2008-2009 lambda-cyhalothrin was used in combination with mass screening and treatment as a combined intervention aimed at district-wide elimination [10].

In 2009, both DDT and pyrethroid resistance were detected at IRS districts [11] and the IRS programs switched to spraying with carbamate (Bendiocarb) [12].
Epidemiological impacts were few, however, at Apac district a $45 \%$ reduction in community parasite prevalence in children under five years was attributed to IRS using DDT (2007-08) and Bendiocarb (2009-10) [12], and a $62 \%$ reduction in health service use for malaria morbidity was associated with Bendiocarb IRS [13].

Between April and October 2014, IRS was scaled down in the northern districts (Acholi sub-region) and switched to seven mid-northern districts (Lango subregion: Alebtong, Amolator, Dokolo, Kabaremaido, Lira, Otuke) and eight eastern districts (Serere, Pallisa, Kibuku, Budaka, Namutumba, Butaleja, Bugiri, Tororo) initially using Bendiocarb and later switching to Actellic 300 CS (organophosphate pirimiphos-methyl). Cessation of IRS in the Northern Districts resulted in large-scale epidemics from April 2015 through to 2016 affecting over 1 million people [14]. At Apac, 18 months following cessation of IRS, malaria morbidity had returned to pre-IRS baseline levels [15].

Unlike the introduction of IRS during the late 1950s, the rapid expansion of IRS in Uganda from 2007 was not accompanied by a comprehensive, longitudinal epidemiological impact analysis plan across all districts where IRS was implemented [6]. There has been one notable exception involving detailed parasitological, clinical and entomological studies at Nagongera sub-County in Tororo district since 2007 [16-22]. These studies provided conclusive evidence that with $>80 \%$ LLIN coverage and four rounds of IRS (coverage ranging between 85-100\%), the incidence of malaria among children was reduced by $92 \%$ and the prevalence of infection by $93 \%$ $[20,22]$. No studies have been undertaken to assess the impact of IRS on child survival or hospitalization in Tororo District. Here, temporal monthly data have been assembled from a hospital surveillance system at Tororo district hospital to examine the impact of vector control approaches on paediatric malaria infections admissions and their age patterns since 2012 to 2019.

\section{Methods}

\section{Study area and malaria control activities}

Tororo District is in Bukedi sub-region, a historically highly malaria endemic, rural area of eastern Uganda bordering Kenya. The 2014 national census put the population of Tororo District at 517,082 [23]. The area supported an estimated entomological inoculation rate of 310 infective bites per person per year in 2011-12, and children aged under 10 years could expect between $2-3$ clinical events per year with prevalence of community 


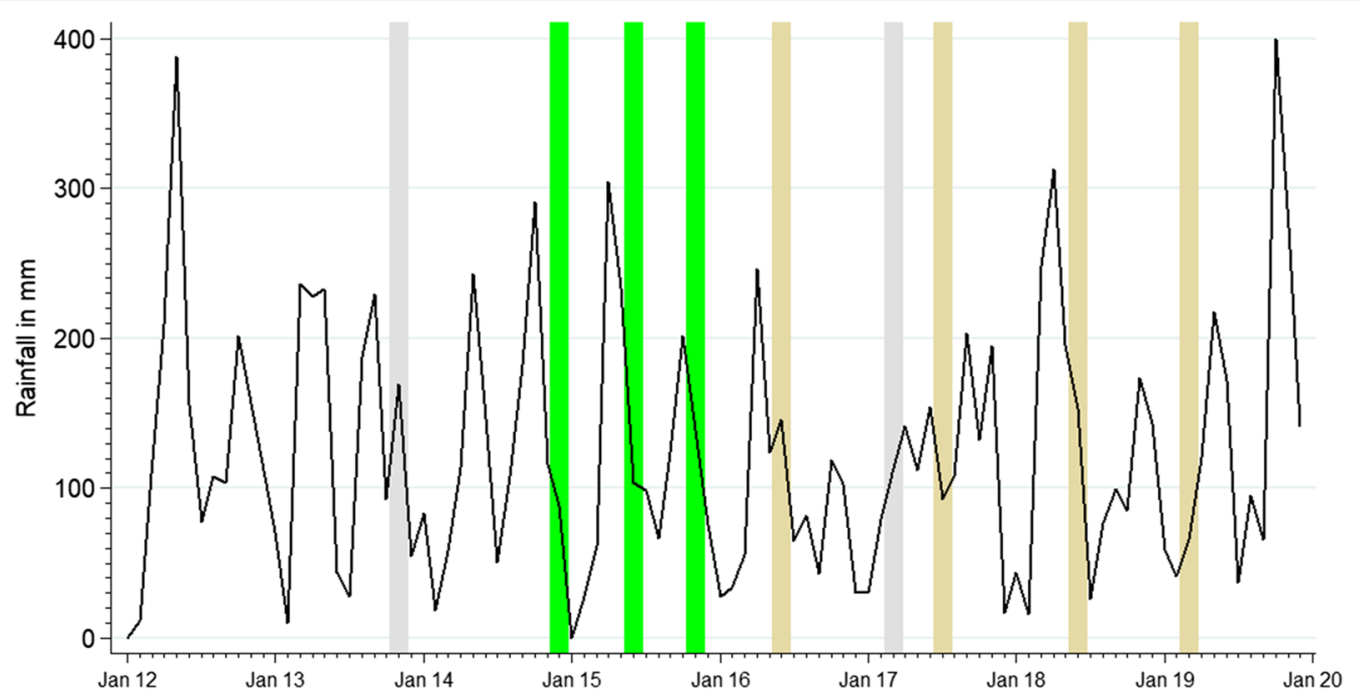

Fig. 1 Rainfall pattern (black line) and distribution of vector control interventions in Tororo district 2012 to 2019. Vertical grey bars represent two rounds of mass distribution of long lasting insecticidal nets. The green bars show the timing of 3 rounds of IRS with Bendiocarb, brown bars 4 rounds of Actellic

infections in this age group $>35 \%$ [24]. Malaria transmission is perennial, however there are two annual peaks coincidental with the two rainy seasons (March-May and August-October) (Fig. 1). Prior to 2014, malaria transmission was maintained predominantly by Anopheles gambiae sensu stricto (s.s.) (82\%) and Anopheles arabiensis (19\%) [25]. Post-2014 following IRS, An. arabiensis (99.5\%) became the dominant vector [19].

The public health care system in Tororo district covers four tiers of out-patient services including 61 level II and III health centres ( $\mathrm{HCs}$ ) providing the most basic services and three level IV HCs providing more advanced levels of care in the form of some in-patient care. There are two hospitals providing general in-patient care, including the main Tororo district hospital with 52 inpatient paediatric beds. The fee-paying St Antony's mission hospital, also located in Tororo town, admits few patients, and does not serve as a major competitor to the government hospital. The nearest Regional Referral Hospital is at Mbale, $46 \mathrm{~km}$ south of the district. Since 2006, artemether-lumefantrine (AL) has been used as the first-line treatment for uncomplicated malaria in Uganda. In 2011, artesunate-amodiaquine was introduced as an alternative first-line treatment to AL [26]. Since 2011, the national standard treatment guidelines have promoted the parasitological diagnosis of malaria at all levels of the health sector, through either rapid diagnostic tests (RDTs) or microscopy [26].

The Government of Uganda has maintained routine distributions of long-lasting insecticide-treated nets (LLIN) since 2007 through antenatal clinics [27]. Mass campaigns of household distributions of LLIN were undertaken in May 2010, November 2013, and June 2017 (Fig. 1). In August 2011, it was estimated that 42\% of the total population were protected by a LLIN [28]; in December 2014, 65\% of the total population were reported sleeping under a LLIN the night before the survey [29]; declining to 46\% in October 2016 [30]; and rising again to $69 \%$ in January 2019 of the sampled population of Tororo [31].

IRS began in Tororo district in December 2014-February 2015 and was repeated in June-July 2015, and November-December 2015 using Bendiocarb (Fig. 1). As a result of resistance to carbamates, annual IRS was switched to the long-lasting organophosphate pirimiphos-methyl (Actellic) from June 2016 [19, 32] (Fig. 1); with studies showing that the main malaria vector, $A n$. gambiae sensu lato (s.l), has been $100 \%$ susceptible to Actellic [33]. In November 2019, 99\% of school children included in a malaria survey in the district reported living in houses that had been sprayed in the last 12 months [34].

\section{Hospital surveillance}

The present study was undertaken using secondary, retrospective analysis of surveillance data at Tororo district hospital. Data were collected as part of an inpatient surveillance program that was initially set up for surveillance of inpatient malaria, but later expanded to surveillance of Acute Febrile Illness (AFI). Details of the programme are described elsewhere [34-36]. In brief, a structured Medical Record Form (MRF) was used to capture standardized 
residential, medical history, clinical examination, and laboratory results on all admissions. Since its inception the surveillance program has supported malaria diagnostic testing (microscopy or rapid diagnostic testing) and emphasized good medical record keeping for all hospitalized children.

Analysis was limited to children resident in Tororo district at the time of hospitalization between January 2012 and December 2019. To determine the physical location of individual households, relevant address information (village, parish, sub-county, and district) recorded on patient MRFs was used to map residences of all admissions to the hospital. Where insufficient information was available on the MRF, missing address details were crosschecked against original hard copy admission registers. If residential information was still missing, admissions were excluded from analysis. Age was calculated in months from reported/documented date of birth and admission date. Neonates were excluded from the analysis and therefore analysis was restricted to children aged 1 month to 14 years.

\section{Data entry and analysis}

Between 2012 and 2015, complete MRFs were entered into an Access database (Microsoft Corporation, Redmond, WA, USA) and transmitted to a central database located in Kampala, Uganda on a monthly basis. From 2016, the data management system was upgraded to a web-based data management system hosted on the District Health Information System (DHIS-2), allowing for real time data entry. Data were analysed using STATA (version 14; STATA Corp., College Station, TX, USA). Monthly malaria test positivity rate (TPR) measured over time formed the basis of the primary analysis and was defined as the number of hospitalized children with a fever (temperature $\geq 37.5{ }^{\circ} \mathrm{C}$ and/or a history of fever in the presenting illness) and a positive malaria test result (RDT or microscopy) among all hospitalized febrile children with a malaria test result. The period of observation ranged from January 2012 to December 2019 and was divided into four time-periods. First, the baseline period, starting January 2012 to October 2013, characterized by routine malaria control interventions. Second, from November 2013 to November 2014, representing the period immediately after the first universal LLIN campaign and before the start of IRS in the district. Third, from December 2014 to May 2016, representing a period of three rounds of IRS with bendiocarb, implemented at six monthly intervals. Fourth, from June 2016 to December 2019, representing four rounds of IRS with Actellic, implemented annually, with a second universal LLIN distribution campaign in the district. Two analytical approaches were used to determine the impact of interventions on TPR during the observation period; beforeand-after analysis and interrupted time series analysis (ITSA).

For before-and-after analysis, the impact of interventions on TPR was assessed by calculating the absolute difference between the aggregated baseline TPR (before) with the three intervention intervals (after): (1) first universal LLIN campaign, (2) IRS with Bendiocarb, and (3) IRS with Actellic and second LLIN campaign. The chi square test was used to assess if differences in TPR were statistically significant. The significance level, alpha, was set at 0.05 . The $95 \%$ CI was determined based on the absolute difference in TPRs plus or minus the margin of error; a product of the standard deviation of the difference between periods and the critical value $(Z=1.960)$. To explore more immediate effects of interventions on TPR, data were also repeated limited to children aged 1-11 months.

ITSA [37-40] was used to assess the effect of IRS on TPR during the observation period January 2012 to December 2019, based on monthly changes in TPR as opposed to aggregated period TPR. The aim was to examine the impact of IRS on monthly-adjusted TPR values during sustained periods of LLIN distribution and use. Therefore, the two observation periods were divided into (a) the first, pre-IRS intervention period starting January 2012 to December 2014 combining the baseline and first LLIN campaign period; and (b) the post-IRS intervention period starting January 2015 to December 2019 combining IRS (Bendiocarb and Actellic) with the second LLIN campaign periods. The end month of the first Bendiocarb IRS campaign was used to reflect the time when implementation of the intervention was completed. To model the effect of IRS on TPR, ordinary least squares regression was used with Newey West standard errors to handle autocorrelation with a 1-month lag. To adjust for possible confounding, models were adjusted for estimates of monthly rainfall in millimetres (recorded at Tororo town) with a 1-month lag, mean age (months) and type of malaria test done (the percentage of all tests done that were RDT). The model estimated the immediate effect of Bendiocarb on TPR, the monthly rate of change in TPR during the pre-intervention period, and the difference between the pre- and post-intervention trends.

Age (months) of hospitalized children with malaria was summarized as median (inter quartile range) and mean ( \pm standard deviation) for the baseline period and different intervention periods stratified depending the malaria test result. A Kruskall-Wallis test was used to test for differences between age during different intervals. The linear temporal change in mean age among test positive 
hospitalized were estimated using linear regression adjusted for 1 month lagged rainfall and type of test done.

\section{Results}

\section{Characteristics of the study population}

Across the 96 months of observation, January 2012 to December 2019, 33,643 admissions aged $\geq 1$ month to 14 years were recorded at Tororo District hospital. 1553 (4.6\%) admissions were residents of neighbouring districts or Kenya, 194 (0.6\%) children had insufficient address information to define district of residence. Among the 31,896 hospitalized children aged 1 month to 14 years resident in Tororo District, 28,049 (90\%) had a fever defined as a temperature $\geq 0.5{ }^{\circ} \mathrm{C}$ or reported history of fever, and 25,884 (93\%) of these children had a malaria test performed at, or during, admission (Additional file 1: Table S1). Overall, among children with fever the TPR was $48.3 \%$, range $19.1 \%$ to $68.6 \%$ over the eight years of observation. TPR decreased from $58.5 \%$ in 2012 to $28.3 \%$ in 2019 , and in children aged 1 to 11 months from $53.6 \%$ to $15.4 \%$. The mean and median ages of children with a positive malaria test results increased over time (Additional file 1: Table S1).

\section{Before and after comparison of TPRs}

Compared to the baseline TPR (60.3\%), TPR was higher during the first LLIN campaign period (67.3\%, difference 7.0\%; 95\% CI 5.2\%, 8.8\%, p <0.001), but was lower during the Bendiocarb IRS (43.5\%, difference $-16.8 \%$; $95 \%$ CI $-18.7 \%,-14.9 \%, \mathrm{p}<0.001)$ and Actellic IRS $(31.3 \%$, difference $-29.0 \%$; 95\% CI $-30.3 \%,-27.6 \%, \mathrm{p}<0.001$ ) (Table 1). Compared to the first LLIN campaign period TPR (67.3\%), TPR decreased during the Bendiocarb IRS period (43.5\%, difference $-23.8 \%$; $95 \% \mathrm{CI}-26.0 \%$, $-21.6 \%, p<0.001)$. Finally, compared to the first LLIN campaign and Bendiocarb IRS period, TPR was lower during the Actellic IRS period combined with the second LLIN campaign (31.3\%, difference - 36.0\%; $95 \%$ CI $-37.7 \%,-34.2 \%, \mathrm{p}<0.001$; difference $-12.2 \%$; $95 \%$ CI $-14.0 \%,-10.3 \%, \mathrm{p}<0.001$, respectively) (Table 1 ). A similar pattern was observed when analysis was limited to children aged 1-11 months, however the difference following the switch from Bendiocarb to Actellic IRS showed a more dramatic decline $(34.3 \%$ vs. $16.6 \%$ difference $-17.7 \%$; $95 \%$ CI $-21.0 \%,-14.4 \%, \mathrm{p}<0.001$ ) (Table 1).

Table 1 Test positivity rate among hospitalized children at Tororo district hospital during different time periods: 2012 to 2019

\begin{tabular}{|c|c|c|c|c|c|}
\hline \multirow[t]{3}{*}{ Variable } & \multirow[t]{3}{*}{ Unit } & \multicolumn{4}{|l|}{ Period } \\
\hline & & \multirow{2}{*}{$\begin{array}{l}\text { Baseline } \\
\text { January } 2012 \\
\text { To October } \\
2013\end{array}$} & \multirow{2}{*}{$\begin{array}{l}\text { 1st mass } \\
\text { LLIN } \\
\text { campaign } \\
\text { November } \\
2013 \text { To } \\
\text { November } \\
2014\end{array}$} & \multirow{2}{*}{$\begin{array}{l}\text { IRS campaign with Bendiocarb } \\
\text { December } 2014 \text { to May } 2016\end{array}$} & \multirow{2}{*}{$\begin{array}{l}\text { IRS with Actellic and 2nd } \\
\text { mass LLIN campaign } \\
\text { June } 2016 \text { to December } 2019\end{array}$} \\
\hline & & & & & \\
\hline Total admissions & $N$ & 9956 & 4712 & 4784 & 12444 \\
\hline \multirow[t]{2}{*}{ Admissions with fever } & $\mathrm{n} / \mathrm{N}$ & 9293/9916 & $4154 / 4422$ & $3897 / 4683$ & $10705 / 12142$ \\
\hline & $\%$ & $93.7 \%$ & $93.9 \%$ & $83.2 \%$ & $88.1 \%$ \\
\hline \multirow{2}{*}{$\begin{array}{l}\text { Admissions with fever tested for } \\
\text { malaria }\end{array}$} & $\mathrm{n} / \mathrm{N}$ & $9073 / 9239$ & $3771 / 4154$ & $3599 / 3897$ & $9441 / 10705$ \\
\hline & $\%$ & $97.6 \%$ & $90.8 \%$ & $92.3 \%$ & $88.1 \%$ \\
\hline \multirow[t]{2}{*}{ TPR among tested with fever } & $\mathrm{n} / \mathrm{N}$ & $5469 / 9073$ & $2537 / 3771$ & $1565 / 3599$ & 2955/9441 \\
\hline & $\%$ & $60.3 \%$ & $67.3 \%$ & $43.5 \%$ & $31.3 \%$ \\
\hline \multirow{2}{*}{$\begin{array}{l}\text { TPR among infants tested with } \\
\text { fever }\end{array}$} & $\mathrm{n} / \mathrm{N}$ & $1614 / 2932$ & $630 / 1090$ & $341 / 994$ & $369 / 2226$ \\
\hline & $\%$ & $55.0 \%$ & $57.8 \%$ & $34.3 \%$ & $16.6 \%$ \\
\hline \multirow{2}{*}{$\begin{array}{l}\text { Fever positives aged } 1 \text { to } \\
11 \text { months }\end{array}$} & $\mathrm{n} / \mathrm{N}$ & $1614 / 5469$ & $630 / 2537$ & $341 / 1565$ & $369 / 2955$ \\
\hline & $\%$ & $29.5 \%$ & $24.8 \%$ & $21.7 \%$ & $12.5 \%$ \\
\hline \multirow[t]{2}{*}{ Fever positives aged 5 to 14 years } & $\mathrm{n} / \mathrm{N}$ & $469 / 5469$ & $261 / 2537$ & $220 / 1565$ & $670 / 2955$ \\
\hline & $\%$ & $8.6 \%$ & $10.3 \%$ & $14.0 \%$ & $22.7 \%$ \\
\hline \multirow[t]{2}{*}{ Age (months) for fever positives } & Median (IQR) & $17(10,29)$ & $18(12,36)$ & $22(12,36)$ & $30(17,48)$ \\
\hline & Mean (SD) & $23.7(21.6)$ & $26.7(24.7)$ & $29.6(25.3)$ & $39.0(30.9)$ \\
\hline
\end{tabular}



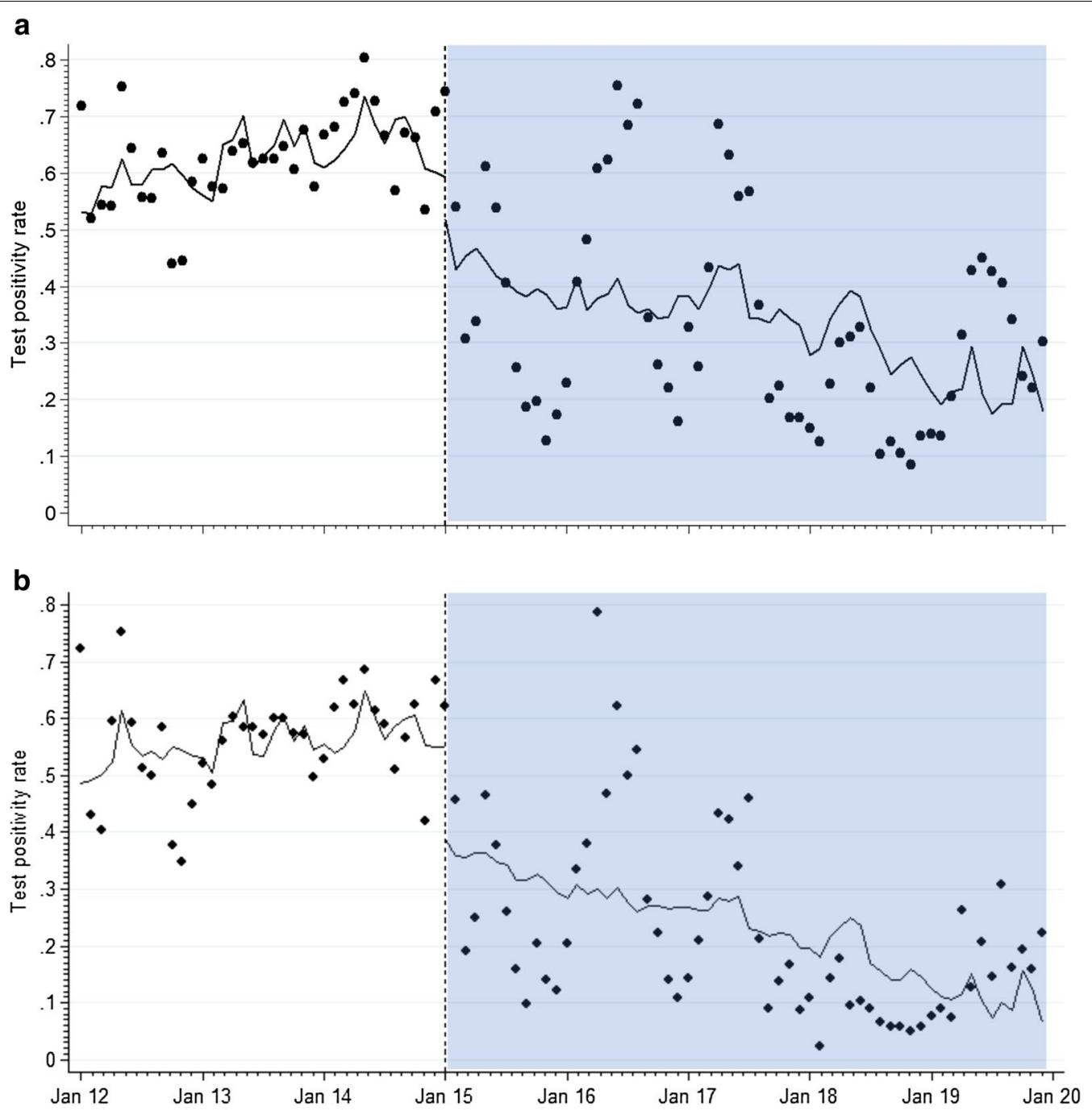

Fig. 2 a Interrupted time series analysis of TPR with Newey-West standard errors and one lag for test positivity rates in relation to initiation of IRS with Bendiocarb (vertical line) in January 2015 among all hospitalized children. The shaded area represents the post-IRS intervention period; IRS (Bendiocarb and Actellic) combined with the second LLIN campaign periods. The pre-IRS intervention period (January 2012 to December 2014) is unshaded (white); the baseline period combined with first LLIN period. b Same as (a), but limited to infants aged 1 to 11 months

\section{Interrupted time series analysis: effect of IRS on monthly changes in TPR}

During the pre-IRS period; combined baseline and first LLIN period (January 2012 to December 2014), monthly rainfall, age and test type adjusted TPRs among children aged 1 month-14 years did not change significantly (Fig. 2a). Immediately after the first Bendiocarb IRS campaign, there was a significant drop in the adjusted TPR by $-14.8 \%$ (95\% CI $-25.6 \%,-2.3 \%, p=0.022$ ) the month after, followed by a decline in the adjusted TPR at a monthly rate of $-0.6 \%$ (95\% CI $-0.9 \%, 0.2 \%$, $\mathrm{p}=0.005$ ) during the combined IRS (Bendiocarb + Actellic) and the second LLIN campaign periods. This trend in the monthly decline of TPR post-IRS was significantly different from the monthly TPR trends during the preIRS period (baseline + first LLIN campaign) (difference in trends $-0.6 \%$, 95\% CI $-1.0 \%,-0.1 \%, \mathrm{p}=0.024$ ) (Fig. 2a). Similar trends post-IRS, without adjustment for age, were noted when analysis was restricted to children aged 1 to 11 months ( $p=0.005$ ) (Fig. 2b).

\section{Changes in age of test-positive admissions}

The monthly mean age of febrile paediatric admissions with a positive malaria test result steadily increased through the baseline and intervention periods $(\mathrm{p}=0.001)$; lowest during the baseline period; median 17 months (IQR 10, 29), mean 23.7 months (SD 21.6) and highest 


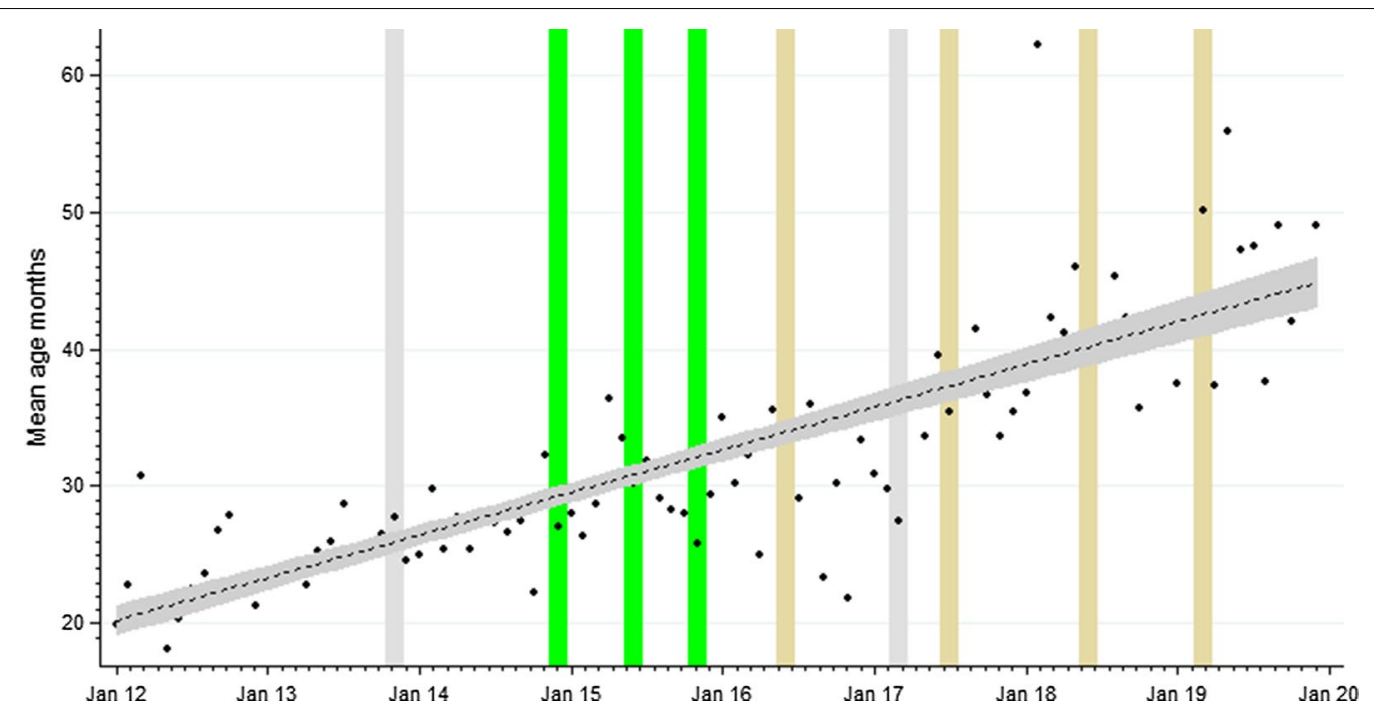

Fig. 3 Monthly trends in the mean age (months) of hospitalized children with positive malaria test result at Tororo district hospital January 2012 to December 2019. A linear best fit line shown as a dashed line. Credible confidence represented as $95 \% \mathrm{Cl}$ (area shaded grey). Interventions represents as shown in Fig. 1

during the last intervention period of IRS with Actellic; median 30 months (IQR 17, 48), mean 30 months (SD 30.9). The regression analysis, adjusting for lagged rainfall and test type showed a significant monthly increase from January 2012 to December 2019 in the mean age of febrile test-positive admissions (Fig. 3: coefficient 0.265, $\mathrm{p}=0.001)$.

\section{Discussion}

The analysis of fever malaria test-positivity rates at Tororo District Hospital from January 2012 to December 2019 highlights significant declines associated with the introduction of IRS in the district (Table 1; Fig. 2). Prior to the introduction of Bendiocarb IRS in December 2014, during a period when the only form of vector control was routine or mass delivery of LLIN, the average TPR among febrile hospital admissions was 63\% (Table 1). However, TPR declined during the periods of IRS use to 43\% (Bendiocarb) and 31\% (Actellic) (Table 1). Interrupted timeseries analysis, controlling for age, rainfall patterns and type of test, shows that compared to the pre-IRS period, IRS (Bendiocarb and Actellic) with the second LLIN campaign period resulted in significant overall monthly reductions in TPR (Fig. 2). Importantly, in this setting of historically high transmission the results demonstrate the combined added value of IRS and LLIN, compared to LLIN alone, findings supported across different settings in Africa [18, 41, 42].

The findings presented here correspond with longitudinal studies of community infection prevalence
2007-2012 (Pre-IRS) and 2014-2017 (Post-IRS, without chemoprophylaxis) among a cohort of children aged 6-30 months in Nagongera sub-county, Tororo [20] and the dramatic reduction in the incidence of clinical malaria in the same cohort [21,32]. A comparison with the patterns of out-patient TPR at Nagongera Health Centre [18, 43], and the hospital TPR (Table 1) showed identical patterns of change.

Throughout the time-series the mean age of paediatric febrile admission malaria test positives increased from 24 months in 2012-2013 to 46 months in 2019 (Fig. 3; Table 1; SI Table 1). Among test-positives during the baseline period (January 2012-October 2013), 29.5\% were aged 1-11 months and $8.6 \%$ were aged 5-14 years, conversely during the last period of Actellic IRS (June 2016-December 2019), 12.5\% were aged 1-11 months and $22.7 \%$ were $5-14$ years (Table 1 ). Changing age-patterns of TPR have also been shown among febrile, paediatric out-patient attendees associated with sustained vector control elsewhere in Uganda [43] and Mozambique [44]. The majority of febrile admissions associated with malaria infection continue to be aged $<5$ years (87\%), however the changing age-pattern among young children suggests a possible impact of sustained, high coverage vector control on the acquisition of immunity among childhood populations [45].

Despite the entomological inoculation rate in Nagongera sub-county declining from 129 infective bites per person per year in 2013 to an apparent zero in 2017 [19], findings from this study suggest that transmission has clearly not been interrupted in the district. During 
the last period of Actellic IRS (June 2016 to December 2019), 31\% of children with fever and tested for malaria presented to the district hospital still harboured malaria infections (Table 1). Limiting the data to all admissions aged 1-11 months, reflecting more recent community transmission in 2019, 90/857 (10.5\%) were either slide or RDT positive. Malaria therefore continues to be associated with a substantial proportion of hospitalizations in the district, despite high coverage of LLIN and IRS using a currently efficacious residual organophosphate. The experimental addition of monthly chemoprophylaxis in Nagongera appears to accelerate a reduction in transmission when used in combination with IRS [17, 20, 22]. Similar findings have been observed when IRS is combined with Mass Drug Administration (MDA) in Zambia [46] and Mozambique [47]. Combinations of vector and chemoprevention of parasite reservoirs are likely to accelerate reductions in transmission [48]. However, the complete interruption of transmission in Uganda is as challenging today as it was during early experimental studies of IRS and drug-based interventions during the 1960s [3-5]. Furthermore, monthly TPR values varied significantly during periods of IRS (Fig. 2), suggesting variations with time since spraying but importantly that any cessation of IRS in Tororo is likely to rapidly result in resurgence of infections among paediatric admissions. Uganda has ample evidence of the dramatic, immediate impact of removing intensive vector control though IRS on out-patient and in-patient hospital burdens [14, 15]. Relying only on moderate-to-high LLIN coverage alone will not mitigate this potential change in malaria burdens.

The WHO recommends that IRS or universal LLIN coverage should be selected as district-wide vector control, not both [49]. The available controlled trial evidence suggests little additional gains of combined LLIN and IRS as compared to the effective implementation of either approach independently [49]. However, in practice national malaria programmes, such as Uganda, continue with LLIN distribution in areas selected for IRS with the benefit of mitigating insecticide resistance [49]. The combination of IRS and LLINs had greater impact on TPR than LLIN used in isolation (Fig. 2; Table 1). This may have arisen because of LLIN and IRS campaigns used different classes of insecticides during a phase of emerging pyrethroid resistance [50, 51]; LLIN distributions have never achieved $100 \%$ coverage in Tororo [31], high bed net attrition rates [52], and those who own LLINs might not always use them [53]. Importantly, as shown in Uganda, sustaining IRS is heavily dependent on donor support, and when donors elect to change support for IRS, or the location, it is essential that these communities continue to be protected by LLIN.
The use of fever TPR has avoided the attribution of admissions to a specific admission diagnosis of malaria, in part because admissions have multiple causes, but largely because fever surveillance for infection is a much simpler, less ambiguous surveillance outcome. Unlike routine health information systems in Uganda which provide only crude age groupings above and below 5 years of age, it has been possible in the hospital analysis presented here to explore in more detail the precise age of test positives with time. However, long-term hospital surveillance suffers from several intrinsic problems that require contextualization, for example changing diagnostic protocols, access to diagnostics and patient access. Furthermore, TPR is less sensitive to measuring the true magnitude of the impact of interventions as it ranges from $0-100 \%$ and cannot represent incidence expressed by the number of people at risk of hospitalization. Defining incidence through routine data, affected by access and use of services, is complex [54] and the relationships between facility based TPR and incidence [55] and community prevalence [56] are non-linear. Nevertheless, routine surveillance of the public health impact of vector control will provide early warnings of a changing disease epidemiology. There is a growing body of evidence that suggests that the impact of IRS can be monitored through health facility and hospital data from routine health information systems in Uganda [13-15, 43, 56-62]. The continued surveillance of the health impact of IRS in Tororo is critical to understand possible further changes in TPR and the age-patterns of malaria admission.

\section{Conclusions}

The findings from this study have important policy implications regarding malaria control in Uganda. The combination of IRS with LLIN distribution has a larger impact than LLIN alone. Sustained vector control increases the mean age of febrile test-positive admissions to hospital a likely result of delayed immune acquisition. However, even after many years of IRS the majority of febrile test positive are still among young children. LLINs and IRS used in combination will not be enough to achieve elimination in Uganda. Combinations of interventions that target vectors and the parasite might be required to interrupt transmission including MDA and vaccines. Routine enhanced health facility-based malaria surveillance data offers a cost-effective means of monitoring the impact of malaria control interventions on malaria infection rates over-time. Expanding the adoption of a standardized MRF for paediatric admissions across the country by the Ministry of Health, and regular interrogation of hospital data will provide insights into intervention impacts, 
failures and an epidemiological understanding of the changing clinical patterns of disease.

\section{Supplementary information}

Supplementary information accompanies this paper at https://doi. org/10.1186/s12936-020-03490-4.

Additional file 1: Table S1. Summary of key indicators among hospitalized children at Tororo hospital: 2012 to 2019.

\section{Abbreviations}

AFI: Acute febrile illness; AL: Artemether-Lumefanthrine; IDI: Infectious Diseases Institute; IQR: Inter-quartile range; IRS: Indoor residual spraying; IQR: Inter quartile range; ITS: Interrupted time series; LLIN: Long-lasting insecticidetreated nets; MDA: Mass drug administration; MRF: Medical record form; $\mathrm{MOH}$ : Ministry of health; NMCP: National Malaria Control Programme; PMI: Presidents Malaria Initiative; RDT: Rapid Diagnostic Test; SD: Standard deviation; TPR: Test positivity rate; UMSP: Uganda malaria surveillance project.

\section{Acknowledgements}

The authors are grateful to the clinical, nursing, and records staff at Tororo hospital for their continued support for the surveillance, notably Thomas Ochar, Stella Agimgi, Rose Osinde, Sabella Abo and Richard Male. We are also indebted to the Uganda Malaria Surveillance Project (UMSP) data officers based at Tororo since 2012: Richard Male, Jospeh Wadamba, and Anthony Ekisa for their commitment to the project, and Proper Bihumuza and the HISP team for development of the web-based data management system. We would also like to thank the IDI team lead by Mohammed Lamorde, Franklin Kizito, and Rodney Mugasha for supporting and sustaining the sites during the AFI program. Allen Okullo and Yasin Kisambira are acknowledged for their help with assembling the rainfall data. Finally, we are grateful to Professor Grant Dorsey for his continued commitment to UMSPs surveillance in Tororo and comments on an earlier draft of the manuscript.

\section{Authors' contributions}

AM and RWS conceived the study; AM analysed the data. LS and DK provided help in spatial location of addresses of the admissions and cleaning of the data. DR and JO provided critical input to the data interpterion. AM and RWS drafted the first version of the manuscript. All authors read and approved the final manuscript.

\section{Funding}

This work was supported through cooperative agreement number 5NU2GGH001744-02-00, funded by the US Centers for Disease Control and Prevention. Its contents are solely the responsibility of the authors and do not necessarily represent the official views of the Centers for Disease Control and Prevention or the Department of Health and Human Services. RWS is funded by Wellcome Trust Principal Fellowship (number 212176), that also provided support for data re-assembly in 2019 and DK. LS is supported by a Wellcome Trust Research Training Fellowship to Dr. Victor Alegana (number 211208). LS, DK and RWS acknowledge the support of the Wellcome Trust to the Kenya Major Overseas Programme (number 203077).

\section{Availability of data and materials}

The datasets used and/or analysed during the current study are available from the corresponding author on reasonable request.

\section{Ethics approval and consent to participate}

The surveillance included the collection of routine hospital data approved as public health surveillance/non-research by CDC (NCEZID \#031416), and approved by the Director General of Health Services, Ministry of Health, Uganda. All data were anonymized without patients or guardian's names prior to analysis.

\section{Consent for publication}

Not applicable.

\section{Competing interests}

The authors declare that they have no competing interests.

\section{Author details}

${ }^{1}$ Child Health and Development Centre, Makerere University, College of Health Sciences, Kampala, Uganda. ${ }^{2}$ Infectious Diseases Research Collaboration, Kampala, Uganda. ${ }^{3}$ National Malaria Control Division, Ministry of Health, Kampala, Uganda. ${ }^{4}$ Department of Prevention, Care and Treatment, Infectious Diseases Institute, Kampala, Uganda. ${ }^{5}$ Population Health Unit, Kenya Medical Research Institute/Wellcome Trust Research Programme, Nairobi, Kenya. ${ }^{6}$ Centre for Tropical Medicine and Global Health, Nuffield Department of Medicine, University of Oxford, Oxford, UK.

Received: 21 August 2020 Accepted: 9 November 2020

Published online: 19 November 2020

\section{References}

1. Oxborough RM. Trends in US President's Malaria Initiative-funded indoor residual spray coverage and insecticide choice in sub-Saharan Africa (2008-2015): urgent need for affordable, long-lasting insecticides. Malar J. 2016;15:146.

2. Tangena JA, Hendriks CMJ, Devine M, Tammaro M, Trett AE, Williams I, et al. Indoor residual spraying for malaria control in sub-Saharan Africa 1997 to 2017: an adjusted retrospective analysis. Malar J. 2020;19:150.

3. De Zulueta J, Kafuko GW, McCrae AWR, Cullen JR, Pedrsen CK, Wasswa DFB. A malaria eradication experiment in the highlands of Kigezi (Uganda). East Afr Med J. 1964;41:102-20.

4. De Zulueta J, Kafuko GW, Cullen JR, Pedersen CK. The results of the first year of a malaria eradication pilot project in Northern Kigezi (Uganda). East Afr Med J. 1961;38:1-26.

5. Najera JA, Shidrawi GR, Gibson FD, Stafford JS. A large-scale field trial of malathion as an insecticide for antimalarial work in Southern Uganda. Bull World Health Organ. 1967;36:913-35.

6. Talisuna AO, Noor AM, Okui AP, Snow RW. The past, present and future use of epidemiological intelligence to plan malaria vector control and parasite prevention in Uganda. Malar J. 2015;14:158.

7. Bukirwa H, Yau V, Kigozi R, Filler S, Quick L, Lugemwa M, et al. Assessing the impact of indoor residual spraying on malaria morbidity using a sentinel site surveillance system in Western Uganda. Am J Trop Med Hyg. 2009;81:611-4.

8. Africa Fighting Malaria (AFM). A Field report of Uganda's Efforts to Build a Comprehensive Malaria Control Program. 2007. https://www.fightingma laria.org/pdfs/AFM_Uganda_Report_9.04.07.pdf.

9. Abt Associates. Uganda Indoor Residual Spraying (IRS) Project Year Two Annual Report, October 1st, 2010 through September 30th, 2011 Uganda Indoor Residual Spraying Project. Massachusetts: Abt Associates Inc; 2011.

10. Yeka A, Echodu D (2018). Impact of population based Indoor Residual Spraying (IRS) in combination with chemotherapy on key malaria indicators in a high transmission setting in north eastern Uganda (Katakwi Rotary Malaria Project -KRMP-). 2018; https://mesamalaria.org/mesa-track /impact-population-based-indoor-residual-spraying-irs-combinatio n-chemotherapy-key.

11. Verhaeghen K, Bortel WV, Roelants P, Okello PE, Talisuna A, Coosemans M. Spatio-Temporal patterns in kdr frequency in permethrin and DDT resistant Anopheles gambiae ss from Uganda. Am J Trop Med Hyg. 2010;82:566-73.

12. Steinhardt LC, Adoke Y, Nasr S, Wiegand RE, Rubahika D, Sserwanga $A$, et al. Effects of indoor residual spraying on malaria and anemia in a high transmission area of Northern Uganda. Am J Trop Med Hyg. 2013;88:855-61.

13. Kigozi R, Baxi SM, Gasasira A, Sserwanga A, Kakeeto S, Nasr S, et al. Indoor Residual Spraying of insecticide and malaria morbidity in a high transmission intensity area of Uganda. PLOS ONE. 2012;7:e42857.

14. Okullo AE, Matovu JKB, Ario AR, Opigo J, Wanzira H, Oguttu DW, et al. Malaria incidence among children less than 5 years during and after cessation of indoor residual spraying in Northern Uganda. Malar J. 2017;16:319. 
15. Raouf S, Mpimbaza A, Kigozi R, Sserwanga A, Rubahika D, Katamba H, et al. Resurgence of malaria following discontinuation of indoor residual spraying of insecticide in an area of Uganda with previously high-transmission intensity. Clin Infect Dis. 2017;65:453-60.

16. Arinaitwe E, Sandison TG, Wanzira H, Kakuru A, Homsy J, Kalamya J, et al. Artemether-lumefantrine versus dihydroartemisinin-piperaquine for falciparum malaria: a longitudinal, randomized trial in young Ugandan children. Clin Infect Dis. 2009;49:1629-37.

17. Bigira V, Kapisi J, Clark TD, Kinara S, Mwangwa F, Muhindo MK, et al. Protective efficacy and safety of three antimalarial regimens for the prevention of malaria in young Ugandan children: a randomized controlled trial. PLoS Med. 2014;11:e1001689.

18. Katureebe A, Zinszer K, Arinaitwe E, Rek J, Kakande E, Charland K, et al. Measures of malaria burden after long-lasting insecticidal net distribution and indoor residual spraying at three sites in Uganda: a prospective observational study. PLoS Med. 2016;13:e1002167.

19. Musiime AK, Smith DL, Kilama M, Rek J, Arinaitwe E, Nankabirwa J, et al. Impact of vector control interventions on malaria transmission intensity, outdoor vector biting rates and Anopheles mosquito species composition in Tororo. Uganda Malar J. 2019;18:445.

20. Kamya MR, Kakuru A, Muhindo M, Arinaitwe E, Nankabirwa JI, Rek J, et al. The impact of control interventions on malaria burden in young children in a historically high-transmission district of Uganda: a pooled analysis of cohort studies from 2007 to 2018. Am J Trop Med Hyg. 2020;103:785-92.

21. Nankabirwa Jl, Briggs J, Rek J, Arinaitwe E, Nayebare P, Katrak S, et al. Persistent parasitemia despite dramatic reduction in malaria incidence after 3 rounds of Indoor Residual Spraying in Tororo Uganda. J Infect Dis. 2019;219:1104-11.

22. Nankabirwa JI, Arinaitwe E, Rek J, Kilama M, Kizza T, Staedke SG, et al. Malaria transmission, infection, and disease following sustained indoor residual spraying of insecticide in Tororo. Uganda Am J Trop Med Hyg. 2020;103:20-0250.

23. Uganda Bureau of Statistics (UBOS). National Population and Housing Census 2014-Main Report. Kampala, 2014.

24. Kamya MR, Arinaitwe E, Wanzira H, Katureebe A, Barusya C, Kigozi SP, et al. Malaria transmission, infection, and disease at three sites with varied transmission intensity in Uganda: implications for malaria control. Am J Trop Med Hyg. 2015;92:903-12.

25. Maxwell K, Smith DL, Hutchinson R, Kigozi R, Lavoy G, et al. Estimating the annual entomological inoculation rate for Plasmodium falciparum transmitted by Anopheles gambiae s.l. using three sampling methods in three sites in Uganda. Malar J. 2014;13:111.

26. Uganda National Malaria Control Division (NMCD), Ministry of Health. 2011

27. Kolaczinski JH, Kolaczinski K, Kyabayinze D, Strachan D, Temperley $\mathrm{M}$, Wijayanandana $\mathrm{N}$, et al. Costs and effects of two public sector delivery channels for long-lasting insecticidal nets in Uganda. Malar J. 2010;9:102.

28. Uganda Bureau of Statistics (UBOS) \& ICF International Inc. Uganda Demographic and Health Survey 2011 Kampala, Uganda: UBOS and Calverton. Maryland: ICF International Inc; 2011.

29. Uganda Bureau of Statistics (UBOS) \& ICF International. Uganda Malaria Indicator Survey 2014-15. Kampala: UBOS and ICF International; 2015.

30. Uganda Bureau of Statistics (UBOS) and ICF. Uganda Demographic and Health Survey 2016. Kampala: UBOS and ICF; 2018.

31. Uganda Bureau of Statistics (UBOS), and ICF. Uganda Malaria Indicator Survey 2018-2019. Kampala, Uganda, and Rockville, Maryland, USA: NMCD, UBOS, and ICF. 2020.

32. Zinszer K, Charland K, Vahey S, Jahagirdar D, Rek JC, Arinaitwe E, et al. The impact of multiple rounds of indoor residual spraying on malaria incidence and hemoglobin levels in a high-transmission setting. J Infect Dis. 2020;221:304-12.

33. Abt Associates. The PMI vectorlink project Uganda 2019 end of spray report Phase I: March 18 - April 15, 2019 and phase II: May 27-June 27, 2019 Uganda Indoor Residual Spraying Project. Massachusetts: Abt Associates Inc; 2019.

34. Mpimbaza A, Walemwa R, Kapisi J, Sserwanga A, Namuganga JF, Kisambira Y, et al. The age-specific incidence of hospitalized paediatric malaria in Uganda. BMC Infect Dis. 2020;20:503.
35. Lamorde M, Mpimbaza A, Walwema R, Kamya M, Kapisi J, Kajumbula H, et al. A Cross-cutting approach to surveillance and laboratory capacity as a platform to improve health security in Uganda. Health Secur. 2018;16(S1):S76-86.

36. Mpimbaza A, Miles M, Sserwanga A, Kigozi R, Wanzira H, Rubahika D, et al. Comparison of routine health management information system versus enhanced inpatient malaria surveillance for estimating the burden of malaria among children admitted to four hospitals in Uganda. Am J Trop Med Hyg. 2015;92:18-21.

37. Lechthaler F, Matthys B, Lechthaler-Felber G, Likwela JL, Mavoko HM, Rika JM, et al. Trends in reported malaria cases and the effects of malaria control in the Democratic Republic of the Congo. PLoS ONE. 2019;14:e0219853.

38. Gilmour S, Degenhardt L, Wayne H, Day C. Using intervention time series analyses to assess the effects of imperfectly identifiable natural events: a general method and example. BMC Med Res Method. 2006;6:16.

39. Linden A. Conducting interrupted time series analysis for single and multiple group comparisons. Stata Journal. 2015;15:480-500.

40. Bernal JL, Cummins S, Gasparrini A. Interrupted time series regression for the evaluation of public health interventions: a tutorial. Int J Epidemiol. 2017;46:348-55.

41. Kleinschmidt I, Schwabe C, Shiva M, Segura JL, Sima V, Mabunda SJ, et al. Combining indoor residual spraying and insecticide-treated net interventions. Am J Trop Med Hyg. 2009;81:519-24.

42. West PA, Protopopoff N, Wright A, Kivaju Z, Tigererwa R, Mosha FW, et al. Indoor residual spraying in combination with insecticide-treated nets compared to insecticide-treated nets alone for protection against malaria: a cluster randomised trial in Tanzania. PLoS Med. 2014;11:e1001630.

43. Kigozi SP, Kigozi RN, Epstein A, Mpimbaza A, Sserwanga A, Yeka A, et al. Rapid shifts in the age-specific burden of malaria following successful control interventions in four regions of Uganda. Malar J. 2020;19:128.

44. Galatas B, Guinovart C, Bassat Q, Aponte JJ, Nhamússua L, Macete E, et al. A prospective cohort study to assess the micro-epidemiology of Plasmodium falciparum clinical malaria in Ilha Josina Machel (Manhiça, Mozambique). Malar J. 2016;15:444.

45. Rodriguez-Barraquer I, Arinaitwe E, Jagannathan P, Kamya MR, Rosenthal PJ, et al. Quantification of anti-parasite and anti-disease immunity to malaria as a function of age and exposure. Elife. 2018;7:e35832.

46. Eisele TP, Bennett A, Silumbe K, Finn TP, Porter TR, Chalwe V, et al. Impact of four rounds of mass drug administration with dihydroartemisininpiperaquine implemented in Southern Province Zambia. Am J Trop Med Hyg. 2020;103(suppl 2):7-18.

47. Galatas B, Saúte F, Martí-Soler H, Guinovart C, Nhamussua L, Simone W, et al. Feasibility of malaria elimination in Southern 1 Mozambique: the Magude project. PLoS Med. 2020;17:e1003227.

48. Alonso PL. The role of mass drug administration of antimalarials. Am J Trop Med Hyg. 2020;103(2_Suppl):1-2.

49. WHO. Guidelines for malaria vector control. Geneva: World Health Organization; 2019.

50. Morgan JC, Irving H, Okedi LM, Steven A, Wondji CS. Pyrethroid resistance in an Anopheles funestus population from Uganda. PLoS ONE. 2010;5:e11872.

51. Okia M, Hoel DF, Kirunda J, Rwakimari JB, Mpeka B, Ambayo D, et al. Insecticide resistance status of the malaria mosquitoes: Anopheles gambiae and Anopheles funestus in eastern and northern Uganda. Malar J. 2018;17:157.

52. Gonahasa S, Maiteki-Sebuguzi C, Rugnao S, Dorsey G, Opigo J, Yeka A, et al. LLIN Evaluation in Uganda Project (LLINEUP): factors associated with ownership and use of long-lasting insecticidal nets in Uganda: a crosssectional survey of 48 districts. Malar J. 2018;17:421.

53. Krezanoski PJ, Santorino D, Agaba A, Dorsey G, Bangsberg DR, Carroll RW. How are insecticide-treated bednets used in Ugandan households? A comprehensive characterization of bednet adherence using a remote monitor. Am J Trop Med Hyg. 2019;101:404-11.

54. Alegana VA, Okiro EA, Snow RW. Routine data for malaria morbidity estimation in Africa: challenges and prospects. BMC Med. 2020;18:121.

55. Boyce RM, Reyes R, Matte M, Ntaro M, Mulogo E, Lin F-C, et al. Practical implications of the non-linear relationship between the test positivity rate and malaria incidence. PLOS ONE. 2016;11:e0152410. 
56. Kamau A, Mtanje G, Mataza C, Mala L, Bejon P, Snow RW. The relationship between facility-based malaria test positivity and community-based prevalence. PLoS ONE. 2020;15:e0240058.

57. Tugume A, Muneza F, Oporia F, Kiconco A, Kihembo C, Kisakye AN, et al. Effects and factors associated with indoor residual spraying with Actellic 300 CS on malaria morbidity in Lira District Northern Uganda. Malar J. 2019;18:44.

58. Tukei BB, Beke A, Lamadid-Figueroa H. Assessing the effect of indoor residual spraying (IRS) on malaria morbidity in Northern Uganda: a before and after study. Malar J. 2017;16:4.

59. Ashton RA, Bennett A, Al-Mafazy AW, Abass AK, Msellem Ml, et al. Use of routine health information system data to evaluate impact of malaria control interventions in Zanzibar, Tanzania from 2000 to 2015. EClin Med. 2019;12:11-9

60. Dokurugu EM, Nasiru S, Majeed BA. The effects of malaria control interventions on out-patient and in-patient malaria cases in the Northern Region of Ghana. Asian J Probabil Stat. 2020;6:61-73.
61. Gogue C, Wagman J, Tynuv K, Saibu A, Yihdego Y, Malm K, et al. An observational analysis of the impact of indoor residual spraying in Northern, Upper East, and Upper West Regions of Ghana: 2014 through 2017. Malar J. 2020;19:242.

62. Wagman J, Gogue C, Tynuv K, Mihigo J, Bankineza E, Bah M, et al. An observational analysis of the impact of indoor residual spraying with non-pyrethroid insecticides on the incidence of malaria in Ségou Region, Mali: 2012-2015. Malar J. 2018;17:19.

\section{Publisher's Note}

Springer Nature remains neutral with regard to jurisdictional claims in published maps and institutional affiliations.
Ready to submit your research? Choose BMC and benefit from:

- fast, convenient online submission

- thorough peer review by experienced researchers in your field

- rapid publication on acceptance

- support for research data, including large and complex data types

- gold Open Access which fosters wider collaboration and increased citations

- maximum visibility for your research: over $100 \mathrm{M}$ website views per year

At BMC, research is always in progress.

Learn more biomedcentral.com/submissions 